\title{
INITIATION AND GROWTH OF EXPLOSION IN SOLIDS
}

A CONSIDERABLE amount of work has been carried out in recent years on slow and fast reactions in solids, and in particular on explosive solids. In order to bring this work together and to review the present position of this field of research, a discussion arranged by Dr. F. P. Bowden for the Royal Society was held on May 30 in the Society's rooms at Burlington House. The discussion was international in character, and there were contributors and visitors from Australia, Belgium, France, Great Britain, Sweden, Switzerland, the United States and the U.S.S.R. The programme was an ambitious one, and by concentrating only on essential points and the more recent advances, it was possible to fit in twenty-two prepared papers. The discussion on these papers was lively and valuable, and many new points were brought out at this stage. There was some time for informal discussion. The papers and the discussion will be published in the Proceedings of the Royal Society.

The discussion fell into two parts. 'The first, on the initiation of explosion, included some work on the slow decomposition of a number of explosive materials. The second part dealt with the growth of reaction to explosion, and with low-velocity detonation. The process of high-velocity detonation was not considered since this had been dealt with in a discussion in 1951 organized by Sir William Penney.

In opening the discussion, Dr. F. P. Bowden summarized the different ways in which reactions in solids may be initiated. 'These are: (1) by heat which raises the material to the ignition temperature; (2) by shock; this can act by $(a)$ an adiabatic heating of compressed gas spaces ; (b) a frictional hot spot on the confining surface or on a grit particle; (c) intercrystalline friction of the explosive itself ; (d) viscous heating of the explosive at high rates of shear ; $(e)$ heating of a sharp point when it is deformed plastically ; $(f)$ mutual reinforcement of gentle shock waves; (3) by ultrasonic vibrations; (4) by electrons, $\alpha$-particles, neutrons and fission fragments; (5) by light of sufficient intensity; (6) by electric discharge; (7) by 'spontaneous' ignition of a growing crystal.

Dr. Bowden emphasized the importance of using modern physical methods to follow reactions in solids. The combined use of electron diffraction and electron microscopy by Dr. J. Sawkill has shown that it is possible to follow the collapse of the lattice during decomposition in materials such as silver azide to give the final product silver. With the aid of the scanning electron microscope, J. A. McAuslan has also followed the early stages in the decom. position of silver azide. There is evidence that reaction can occur preferentially not only on the surface of the crystal but also at defects within it. This leads to a cracking and splitting of the crystals along preferred directions. This break-up has also been observed by S. K. Deb in his experiments on the decomposition of P.E.T.N. crystals by a light flash. The disintegration of crystals into small blocklets, which may be about $10^{-5} \mathrm{~cm}$. across, is important both in the early phase and also, as shown by Evans and Yoffe, in the growth of burning to detonation.

Some puzzling observations made by G. T. Rogers on spontaneous explosions were also discussed.
During the growth of crystals of $\beta$-lead azide and mercuric azide in solution, an explosion may take place for no apparent reason. Dr. J. V. R. Kaufman (United States) said that $\alpha$-lead azide also explodes spontaneously during the initial stages of growth in solution. There is at present no adequate explanation for these observations, although some suggestions have been put forward. Dr. Z. V. Harvalik (United States) thought that the heat of aggregation of small crystals could be an important factor.

Thermal decomposition and explosion. The growth of explosion from the point of initiation is a critical process. If the size of the nucleus of decomposition or 'hot spot' is greater than about $10^{-3} \mathrm{~cm}$. in diameter, then for a number of explosive materials there is a chance that the reaction will grow to explosion. Thermal factors determine this growth, and it is necessary for the rate of heat liberation to be greater than the rate of heat loss. Two problems in thermal explosion theory were analysed by $\mathrm{Dr}$. G. B. Cook. The first was concerned with a slab of solid explosive, where one face is placed in contact with a constant-temperature bath. From his calculations, he obtained a relation between ignition time and the temperature of the bath and the initial temperature of the explosive. The interesting point that emerges is that for a given initial temperature the order of thermal sensitivity can change with the bath temperature. The examples given by Dr. Cook are 'Tetryl', P.E.T.N. and R.D.X.

A paper on the thermal decomposition of silver azide by Drs. B. E. Bartlett, F. C. Tompkins and D. A. Young was read by Dr. F. C. Tompkins. The decomposition was followed both above and below the crystallographic transformation at $190^{\circ} \mathrm{C}$. Dr. Tompkins stressed the importance of the interfacial reaction at the product-reactant interface. Activation energies for the reaction were given. Below $190^{\circ} \mathrm{C}$. this is $44 \mathrm{kcal}$./mole and above that point it is $31 \mathrm{kcal}$./ mole. These authors also presented some results on the ionic and photoconductance of silver azide.

Initiation by shock. Three papers were presented on the ignition of explosive materials by the compression of gas spaces. Dr. C. H. Johansson (Sweden) considered the case of liquid materials. He showed that in a number of cases a foam is produced during impact. This means that the disintegration of the liquid into tiny droplets is important. He also discussed other experiments on the initiation of explosion in liquids.

To obtain accurate values for the energy required to ignite a number of sensitive materials, Dr. G. J. Bryan and Dr. E. C. Noonan (United States) and J. I. Evans and Dr. A. M. Yuill (Ardeer) have constructed compression apparatus of advanced design. With this apparatus it is possible to vary the com. pression ratio and the time of compression, and it is also possible to record the light from the burning explosive by means of a photomultiplier. In the apparatus described by Dr. Noonan the gas pressure could also be read as a function of time.

Prof. K. K. Andreev (U.S.S.R.) in a paper entitled "Some Considerations on the Mechanism of Initiation of Detonation in Explosives" gave a résumé of his own and other Russian work. He also emphasized the importance of the disintegration of the solids. In 
particular, he considers that the initiation of detonation is due mainly to the sudden rise in pressure when a suspension of the solid explosive which is formed in the gaseous products of burning explodes. He also discussed Landau's ideas on the effects of turbulence at the burning front of liquids and the mechanism by which suspensions might be formed. The work discussed by Prof. Andreev is relevant both to the initiation of explosion and to the growth mechanism to detonation.

Structure and stability of inorganic azides. The marked difference in the decomposition behaviour of materials such as potassium azide and silver azide has led B. L. Evans to attempt to correlate the explosive sensitivity of these compounds with their crystal structure. $H_{\theta}$ considered the azides and the isoelectronic cyanates, thiocyanates and fulminates. Thus in the case of azides the important factor to consider is the ionization potential of the metal compared with the electron affinity of the azide radical. This determines both the extent to which the valence electron is under the influence of the metal and the unit cell dimensions of the metallic azide. From the results that are available, Evans concludes that the explosive sensitivity is dependent on the ionization potential of the metal forming the azide. The ionization potential of the metal will also determine some of the physical properties such as the melting point, and it can be used to interpret results on photoconduction, thermal decomposition and photochemical decomposition.

Initiation by light. Prof. J. Eggert and his colleagues (Switzerland) found some time ago that a number of sensitive materials could be ignited by a light flash of sufficient intensity. In presenting the Cambridge work, J. A. McAuslan discussed his own experiments on the ignition of lead styphnate, and that of G. T. Rogers on silver azide. A thermal mechanism has been postulated for the initiation of explosion, in which the absorbed light energy is converted into heat in a very thin layer near the surface. In the case of silver azide, however, this assumption has been questioned by B. L. Evans. His results on sensitized ignitions suggest that the primary action of the light is photochemical, but that propagation of reaction is thermal. A necessary condition for this growth to explosion is the formation of a molten layer on the surface. It is in the molten state that there is sufficient mobility of the azide radicals to give rise to explosive reaction.

Dr. A. C. McLaren and Dr. G. T. Rogers described their results on the absorption spectra (visible light and ultra-violet) and photoconduction of single crystals of silver azide. They explained their results on absorption spectra in terms of the formation of excitons. The photocurrent is due to the dissociation of these excitons into free electrons and positive holes. These results support the suggestion, made earlier, that during the ignition of silver azide by light the initiation is photochemical in nature.

There was a vigorous discussion of the work presented in these last two sections. In this discussion Dr. J. W. Mitchell outlined his views on the action of metal particles as electron-traps in silver azide. Dr. P. Gray gave his views on the use of enthalpies of formation in describing the behaviour of azides.

Decomposition and explosion by ionizing radiations. The slow decomposition of a number of materials by X-rays was considered by Dr. J. V. R. Kaufman (United States) and by Dr. J. M. Groocock. The only material which explodes during irradiation is nitrogen iodide. This was discussed by Dr. F. P. Bowden. In particular, he showed how McLaren's results on the explosion of nitrogen iodide by $\alpha$-particles could be shown to be the result of the removal of ammonia from the surface of the nitrogen iodide by the $\alpha$-particles. Dr. Bowden also discussed some recent experiments by Deb, Evans and Yoffe on the use of the radioisotope of silver as a means of introducing cadmium into erystals of silver azide.

The growth of explosion formed the subject of the second part of the discussion. An introductory paper by Dr. A. D. Yoffe on the work in Cambridge showed that the stages following on initiation of reaction are: (1) growth of reaction to an accelerating burning ; (2) transition from burning to low-velocity detonation ; (3) propagation of low-velocity detonation. Very little is known about stage (2). The growth of reaction to burning depends on thermal factors. The differences in behaviour of azides and the more complex materials during this stage can be explained in terms of the differences in the possible modes of decomposition of these materials.

There is no adequate theory to explain the propa. gation of low-velocity detonation in solids. Factors such as the compression of trapped gas, intercrystalline friction and break-up of the solid ahead of the flame front are important. They do not, however, explain all the results that are available. For example, Deb has found that a low-velocity detonation can be propagated through a single crystal of P.E.T.N. containing no obvious air pockets. The formation of a molten or of a vaporized layer is probably important in the growth to explosion and in the setting up of low-velocity detonation.

High-speed photographic techniques were used by Dr. G. P. Cachia and Dr. E. G. Whitbread, by Prof. M. A. Cook (United States), and by Dr. C. W. Lampson. and Dr. R. J. Eichelberger (United States) to follow the sympathetic detonation of solid explosives by shocks propagated across an air-gap or through a metal strip. Detonation in the receptor charge does not always occur at the surface in contact with the metal wafer, but initiation may occur within the body of the receptor. A ciné-film, prepared at the Aberdeen Proving Ground, United States, illustrated this effect. This film was remarkable for its clarity and showed a high degree of technical competence. It was much appreciated by all present.

The detonation of pentolite in water by a remote spherical primer was discussed by Dr. C. F. Winning. Other papers presented were: studies on the oxidation induced by ultrasonic radiation on a solid system of lauryl aldehyde and potassium chlorate by Dr. F. A. H. Rice and Dr. D. Levine (United States); the ignition of primary explosives by electric discharges by Drs. R. M. H. Wyatt, P. W. J. Moore, G. K. Adams and J. F. Summer; some kinetic problems of fast reactions by Dr. R. B. Parlin and Prof. H. Eyring (United States); and lamellar twinning and decomposition in potassium azide and sodium azide by Dr. R. W. Dreyfuss and Dr. P. W. Levy.

This discussion has shown that a good deal is known about the initiation of explosion in solids. In his summing up, Dr. F. P. Bowden said there is sufficient data now available for us to argue about the electronic structure and possible modes of decom. position of a number of materials. There are, however, a number of outstanding problems. We know very little about radiation damage in solid explosives. There is also the question of spontaneous explosions 
in solids. So far as the growth of explosion is concerned we need more experimental results. In particular, there is even a lack of data of quantities such as thermal conductivity and specific heat. The disintegration and break-up of solids ahead of the reaction zone have been stressed by a number of workers. The formation of a molten zone and its importance, both in the growth of explosion and in low-velocity detonation, have been mentioned. With the development of new apparatus, and with the techniques now available, we may expect a rapid advance in the near future on this problem of the growth of reaction to detonation.

\section{A. D. YoFfe}

\section{RECENT ADVANCES IN HIGH POLYMER PHYSICS}

A

CONFERENCE on "Rheology of Elastomers" was held by the British Society of Rheology during May 29-31 at the British Rubber Producers' Research Association, Welwyn Garden City.

Equilibrium properties of rubberlike materials. In an introductory talk, Dr. L. R. G. Treloar (British Rayon Research Association) said that in the main the properties of rubber and of other polymeric materials in the highly elastic or 'rubberlike' state are now well understood. The stress and birefringence of rubber subjected to different types of finite strain at various temperatures are described to a good irst approximation by the 'kinetic theory', a relatively simple molecular theory which treats of the changes of configurational entropy and molecular orientation in a deforming network composed of flexible long-chain molecules. Reviewing the shortcomings of the theory (in the Gaussian approximation), Dr. Treloar said that for simple elongations in the range 150-500 per cent, the discrepancies between the theoretical and observed stress-strain relations have so far not received satisfactory 'molecular' explanation.

A crucial test of the applicability of the kinetic theory to polymeric materials is furnished by the prediction that the rigidity modulus should increase linearly with the absolute temperature and with the concentration of junctions in the network. By using gelatin gels specially prepared to give junctions which are substantially unaffected by temperature changes, P. R. Saunders and A. G. Ward (British Gelatine and Glue Research Association) found that the rigidity modulus did increase linearly with temperature in the range $0^{\circ}-60^{\circ} \mathrm{C}$. and at a rate com. parable with that required by the theory. Data both from gel rigidities and from dilute solution viscosities at different $p \mathbf{H}$ indicated that differences in the rigidities of ordinary gelatin gels must be attributed to differences in concentration and strength of network junctions, rather than to differences in molecular chain 'stiffness'.

Improved techniques for conditioning filaments of natural rubber so as to yield a more accurate attain. ment of thermodynamic equilibrium (required for comparison with the kinetic theory) were described by $\mathbf{P}$. Thirion (Institut Français du Caoutchouc). For filaments held at constant extension in the range 0-30 per cent, reversibility of tension-temperature cycles $\left(20^{\circ}-60^{\circ}\right.$ C.) was attained to within 0.05 per cent. A new derivation was given of Gee's formula for determining the coefficients of change of entropy and internal energy with change of extension at constant volume and temperature. From the data, the corresponding coefficients at constant pressure and temperature were determined with accuracies of the order $0.03 \mathrm{gm} . / \mathrm{cm} .{ }^{2} / \mathrm{deg}$. C. and $10 \mathrm{gm} . / \mathrm{cm} .{ }^{2}$, respectively.

When the individual 'links' of long-chain molecules in a network have anisotropic optical polarizability, the network becomes birefringent when stressed. According to the kinetic theory, which assumes that no crystallization is present, the stress (for simple elongation) should be proportional to the magnitude of birefringence, and the constant of proportionality, or 'stress-optical coefficient', should not depend on the concentration of network junctions. Dr. D. W. Saunders (British Rayon Research Association) presented data obtained at temperatures above the crystal melting points which agreed with both these theoretical predictions in the case of cross-linked natural rubber and gutta percha. For polythene and polymethylene, however, the stress-optical coefficient decreased with increase of junction concentration; this was satisfactorily accounted for by extending the theory to apply to short-chain networks, and the data indicated that these two polymers had comparatively stiff chains. The problem of giving a strict definition of chain stiffness was considered.

'The creation and loss of junctions in a network deformed from its unstressed state were considered in a theoretical paper by Dr. J. Scanlan (British Rubber Producers' Research Association). Tobolsky and co-workers have postulated a form of strainenergy function which would be obtained if the actual network behaved as if it were two independent net. works, each of which obeyed the kinetic theory. Dr. Scanlan showed that, in the case in which junctions are created but none is lost, this postulate can be justified, but in the case in which one junction is lost for every one created (in the deformed state), the postulated strain-energy function is correct in form but contains coefficients which must be corrected. Two arguments were presented-one based on the network theory of James and Guth, and the other on a corrected form of a theory given by Scott and Stein.

Elastic recovery, flow and stress relaxation. A striking demonstration of high elastic recovery in a liquid was given by Dr. A. S. Lodge (British Rayon Research Association), who poured a liquid (containing about 5 per cent aluminium laurate in paraffin) out of a bottle; on sharply cutting the liquid stream with scissors, the upper part of the stream snapped back into the bottle. Dr. Lodge has applied the network theory of James and Guth and the theory of relaxing networks of Green and Tobolsky to the problem of elastic recovery in solutions of long. chain molecules; for the instantaneous recovery following steady laminar shear flow, the latter theory predicts an unexpected expansion in directions normal to the previous flow lines. The suggestion that this might contribute to the diameter increase commonly observed when polymer solutions and melts are extruded from tubes of circular crosssection gave rise to considerable discussion, and alternative explanations, also in terms of elastic recovery, were mentioned by Dr. P. Clegg (Imperial Chemical Industries, Plastics Division). Dr. Clegg's data for molten polythene showed that at low flow 